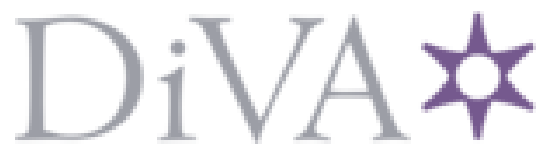

http://www.diva-portal.org

This is the published version of a paper presented at IEEE European Symposium on Security and Privacy Workshops.

Citation for the original published paper:

Dubrova, E., Näslund, O., Degen, b., Gawell, A., Yu, Y. (2019)

CRC-PUF: A Machine Learning Attack Resistant Lightweight PUF Construction

In: 2019 IEEE European Symposium on Security and Privacy Workshops

(EuroS\&PW) (pp. 264-271-). IEEE conference proceedings

N.B. When citing this work, cite the original published paper.

Permanent link to this version:

http://urn.kb.se/resolve?urn=urn:nbn:se:kth:diva-260434 


\title{
CRC-PUF: A Machine Learning Attack Resistant Lightweight PUF Construction
}

\author{
Elena Dubrova Oskar Näslund Bernhard Degen Anders Gawell Yang Yu \\ Royal Institute of Technology (KTH), Stockholm, Sweden \\ \{dubrova, onaslund, bdegen, agawell, yang11\}@kth.se
}

\begin{abstract}
Adversarial machine learning is an emerging threat to security of Machine Learning (ML)-based systems. However, we can potentially use it as a weapon against ML-based attacks. In this paper, we focus on protecting Physical Unclonable Functions (PUFs) against ML-based modeling attacks. PUFs are an important cryptographic primitive for secret key generation and challenge-response authentication. However, none of the existing PUF constructions are both ML attack resistant and sufficiently lightweight to fit low-end embedded devices. We present a lightweight PUF construction, CRC-PUF, in which input challenges are de-synchronized from output responses to make a PUF model difficult to learn. The de-synchronization is done by an input transformation based on a Cyclic Redundancy Check (CRC). By changing the CRC generator polynomial for each new response, we assure that success probability of recovering the transformed challenge is at most $2^{-86}$ for 128-bit challenges and responses.
\end{abstract}

Index Terms-Adversarial machine learning, modeling attack, Physical Unclonable Function (PUF), arbiter PUF.

\section{INTRODUCTION}

Machine Learning (ML) was originally applied in the security domain to detect potential attacks and protect against them [1], [2]. However, sophisticated attacks on cryptographic systems, making use of speed and efficiency of ML followed up quickly [3], [4], [5], [6], [7].

Looking at another side, security of the ML systems is threatened by advances in adversarial machine learning [8], [9], [10], [11]. Adversarial machine learning aims to "fool" an ML system through manipulation of the input data during learning or classification [12], [13]. A natural question that arises: Can we can use the double negative effect (see Fig. 1) and turn adversarial machine learning as a weapon against ML-based attacks?

Physical Unclonable Functions. In this paper, we focus on mitigating ML-based modeling attacks on Physical Unclonable Functions (PUFs). A PUF is a physical instance that generates responses to given input stimuli, called challenges [14], [15]. Due to manufacturing process variation, every transistor or wire in a physical device has slightly different characteristics. These differences lead to measurable differences in responses of two PUF instances to the same challenge, making possible using the PUF as a unique device "fingerprint" [16], [17].

Since process variation cannot be controlled during fabrication of Integrated Circuits (ICs), a hardware clone of a PUF implemented in an IC cannot be made. The responses are generated by the PUF upon request and can thus only be captured by an attacker during a short time interval when the

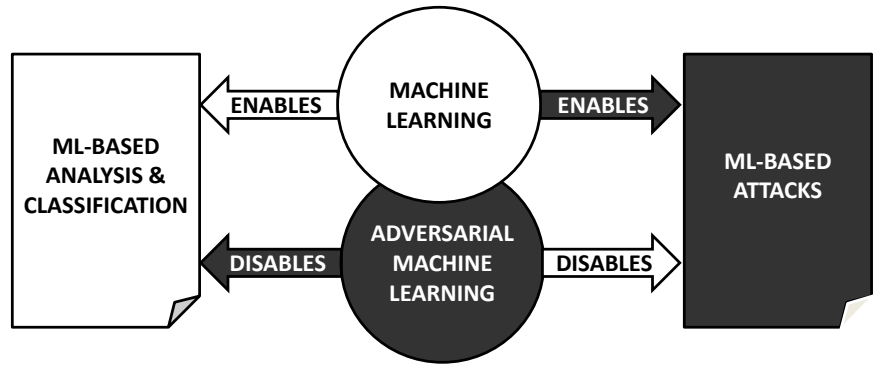

Fig. 1. The double negative effect.

PUF is challenged. Physical attacks on powered-on devices are more difficult than the attacks on power-off devices [18]. These features makes PUFs an ideal cryptographic primitive for challenge-response authentication in applications requiring tamper-resistance. Existing trusted hardware-based methods, such as Apple's secure enclave which prevented the FBI from accessing an iPhone [19], are only cost-efficient for higher-end devices and too expensive for lower-end embedded devices. Software-based methods are usually limited in terms of the assurance they can provide.

Previous Work. Although many different PUFs have been introduced, the search for an efficient and secure PUF continues. The majority of known PUFs are vulnerable to ML-based modeling attacks [3], [20]. To strengthen PUFs' resistance to modeling attacks, different constructions have been presented, including XOR PUFs [21], lightweight PUFs [22], feedforward PUFs [15], interpose PUFs [23], and ising PUFs [24]. However, hardware overhead of these constructions is often too high for resource-constrained devices. It has also been proposed to hash PUF responses using some cryptographically secure hash function [25]. Again, a low-end device such as sensor or actuator, may not have enough storage, computing, and energy resources for implementing a full-strength cryptographic hash function such as SHA-3 [26].

Our Contribution. Inspired by the idea that manipulation of input data can "fool" ML, we propose a new PUF construction, CRC-PUF, which "fools" ML-based modeling attacks by manipulating seed challenges applied to the PUF. The manipulation is performed by a transformation based on a Cyclic Redundancy Check (CRC). By changing the CRC generator polynomial for each response generation session, we assure that the success probability of finding the result of the 


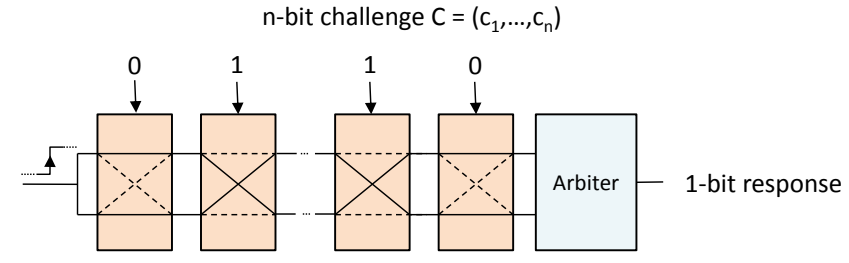

(a)

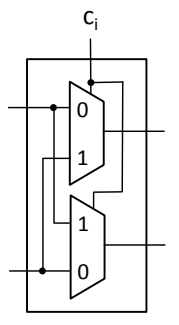

(b)
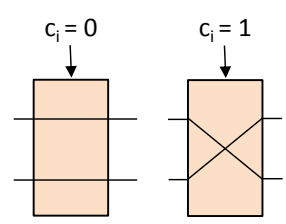

(c)

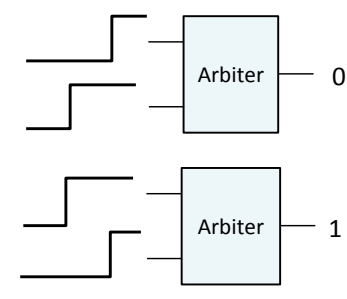

(d)
Fig. 2. (a) Block diagram of an $n$-stage arbiter PUF, (b) switch block implementation, (c) switch block operation, (d) arbiter operation.

transformation is negligibly low.

The construction consists of an $n$-bit Linear Feedback Shift Register (LFSR) with reconfigurable connections and a PUF of type $\{0,1\}^{n} \rightarrow\{0,1\}$. The PUF computes an $m$-bit response $R$ to an $n$-bit seed challenge $C$ as follows. First, $C$ is loaded into the LFSR and the LFSR is clocked $n$ times without producing any output. This is done in order to make the LFSR state unknown to an attacker. Then, $R$ is generated by clocking the LFSR $m$ times and using its state as inputs to the PUF.

Using an LFSR with fixed connections for expanding a seed challenge $C$ into a set of challenges is a common practice in many PUF constructions [27]. It is intended to reduce the amount of data transmitted between a PUF and its verifier. However, to the best of our knowledge, we are the first to propose $n$ void rotations of the LFSR as a means of making inputs to the PUF unknown to an attacker. Void FSR rotations are used in the initialization algorithms of some stream ciphers, e.g. Trivium [28], but, in the stream cipher's case, the FSR connections are known while its starting state (or parts of it) is not. In our case, it is vice versa: the staring state is known, but the LFSR connections are not.

Any PUF of type $\{0,1\}^{n} \rightarrow\{0,1\}$ can be used in the presented construction. We use an arbiter PUF due to its implementation simplicity and good statistical properties.

Paper Outline. The paper is organized as follows. Section II gives the background. Section III describes the presented CRC-PUF construction. Section IV presents security analysis. Section V estimates hardware overhead and Section VI evaluates statistical properties of the CRC-PUFs. Section VII concludes the paper and discusses open problems.

\section{BACKGROUND}

\section{A. Arbiter PUF design}

An $n$-stage Arbiter PUF (APUF) consists of $n$ switch blocks (see Fig. 2). Each switch block $i$, has two inputs, two outputs, and one control input $c_{i}, i \in\{1, \ldots, n\}$. If $c_{i}=0$, straight connections are selected. Otherwise, crossed connections are selected. The response to a challenge $C=\left(c_{1}, \ldots, c_{n}\right)$ is computed by letting two symmetrically designed paths to compete through the chain of switch blocks.

It is easy to see that each different challenge $C \in\{0,1\}^{n}$ selects two different paths out of $2^{n}$ possible paths through the switch blocks chain. An arbiter at the end of the chain determines which of the two paths is faster and generates 1 if the upper path is the "winner", and 0 otherwise. In this way, a "random" Boolean function of $n$ arguments $c_{1}, \ldots, c_{n}$ is induced:

$$
f(C)=\operatorname{sgn}(\Delta(C)),
$$

where $\Delta(C)$ is the delay difference of the upper and lower paths for the challenge $C$ and the $\operatorname{sgn}$ is defined by $\operatorname{sgn}(x)=$ 1 if $x \geq 0$ and $\operatorname{sgn}(x)=0$ otherwise.

The function $f$ is "random" in the sense that we do not know its output values before the PUF is fabricated. Once the PUF is fabricated, the function becomes deterministic (in the error-free case). Small differences in delays caused by manufacturing process variation result in different functions in the individual PUF instances.

\section{B. Arbiter PUF model}

As their name suggests, PUFs should be impossible to clone by physical means. In addition, their responses should be hard to predict by anyone not possessing the PUF, even if responses to many challenges have been seen by an attacker. However, for an $n$-input APUF, there exists a linear additive delay model of size $n+1$ which has the form:

$$
\Delta(C)=\sum_{i=1}^{n+1} w_{i} \Phi_{i}(C)=\langle w, \Phi(C)\rangle
$$

where $w$ and $\Phi$ are the delay vector and the parity (or feature) vector, respectively, defined by

$$
\begin{aligned}
& w_{1}=\delta_{0,1}-\delta_{1,1}, \\
& w_{i}=\delta_{0, i-1}+\delta_{1, i-1}+\delta_{0, i}-\delta_{1, i}, \text { for } 2 \leq i \leq n \\
& w_{n+1}=\delta_{0, n}+\delta_{1, n} .
\end{aligned}
$$

where $\delta_{0, i}$ is the delay difference of straight connections of the switch block $i$ and $\delta_{1, i}$ is the delay difference of crossed connections of the switch block $i$, for $i \in\{1, \ldots, n\}$, and

$$
\begin{aligned}
& \Phi_{i}(C)=\sum_{j=i}^{n}\left(1-2 c_{j}\right), \text { for } 1 \leq i \leq n, \\
& \Phi_{n+1}=1,
\end{aligned}
$$

where the challenge bits $c_{j}$ are encoded as $c_{j} \in\{1,-1\}$.

\section{Modeling attacks on APUF and its extensions}

The simplicity of the APUF's model makes APUFs vulnerable to ML-based modeling attacks. An attacker can collect many challenge-response pairs and use a suitable ML algorithm to create a model for the PUF (2). Once the model is created, the attacker can simulate the APUF's response to any challenge. 
The modeling attacks can be classified into two types [23]: classical, which use random challenges, and reliability-based, which repeat the same challenge multiple times.

1) Classical modeling attacks: The first classical modeling attack on APUF was presented by Lim in his master thesis introducing APUF [21]. He has shown that a 64-bit APUF can be modeled using the Support-Vector Machine (SVM) algorithm. As a countermeasure, Lim proposed $k$-XOR APUF construction, in which responses of $k$ different APUFs operating in parallel are combined by an XOR.

However, in 2010 Ruhrmaier et al. [3] have shown that, for $k \leq 5, k$-XOR APUF can be modeled with the Logistic Regression (LR) algorithm. This result was later expanded by Tobisch and Becker to $k \leq 9$ for 64-bit $k$-XOR APUF and $k \leq 7$ for 128-bit $k$-XOR APUF [20]. For larger values of $k$, $k$-XOR APUFs are considered to be resistant to LR [3]. This is because there exists a sub-exponential relationship between $k$ and the amount of data required to train the model [20]. The same [29] applies to the multiple-output extension of the $k$-XOR APUF, lightweight APUF [22].

The Probably Approximately Correct (PAC) algorithm [30] has been also applied to model $k$-XOR APUFs, but with less success. It is feasible for $k \leq 4$ [31].

Another modification of APUF, feed-forward APUF [15], can be modeled using SVM and LR if the number of feedforward positions is less than 8 [3].

2) Reliability-based modeling attacks: Reliability-based modeling attacks were introduced by Becker in 2015 [32]. Becker's attack can break $k$-XOR APUF with a linear complexity in $k$.

The key idea is that frequency of errors in the response of APUF to a challenge $C=\left(c_{1}, \ldots, c_{n}\right)$ gives us information about the delay differences $\delta_{c_{1}, 1}, \ldots, \delta_{c_{n}, n}$. Close to 0 delay differences cause frequent errors, while larger delay differences result in more stable responses. By applying the same challenge $C$ multiple times, we can evaluate the frequency of errors and then feed the obtained information about $\delta_{c_{1}, 1}, \ldots, \delta_{c_{n}, n}$ to a ML algorithm to learn the model (2). Note that even if APUF is stable under normal environmental conditions, it can usually be made more sensitive to noise by varying temperature or voltage, unless it is protected.

A countermeasure based on repeating the computation of $k$ parallel PUFs $N$ times and applying the majority voting before XORing their responses was suggested in [33]. Note, however, that the reliability of a time redundancy scheme with $N$ repetitions and majority voting is higher than the reliability of a non-redundant module, $R$, only if $R>50 \%$ [34]. Therefore, if a $k$-XOR APUF employing majority voting is exposed to extreme environmental conditions causing each of the $k$ parallel APUFs to fail in more than $50 \%$ of cases, the protected $k$-XOR APUF will fail more often than the unprotected one.

\section{Cyclic Redundancy Check}

Cyclic Redundancy Check (CRC) is widely used for detecting random errors in data communication and storage [35].
Many wireless communication standards, including IEEE 802.15.4, LTE and GSM, use CRC.

Let $G F(2)$ denote a finite field of two elements, $G F(2)=$ $\{0,1\}$. In the sequel, we assume an implicit bijection between the $n$ bit strings $p \in G F\left(2^{n}\right)$ and the polynomials $p(x)=\sum_{i=0}^{n-1} p_{i} x^{i}$ over $G F(2)$, so that the coefficients $\left(p_{0}, \ldots, p_{n-1}\right) \in G F\left(2^{n}\right)$ of $p(x)$ correspond to the bits of $p$.

Given a polynomial $g(x)$ of degree $n$ over $G F(2)$, an $n$-bit $\mathrm{CRC}$ for the message polynomial $M(x)$ is computed as

$$
C R C_{g}(M)=M(x) \cdot x^{n} \bmod g(x) .
$$

The polynomial $g(x)$ is called the generator polynomial of the CRC. The coefficients of $C R C_{g}(M)$ constitute the check bits of the CRC.

The CRC computation can be efficiently implemented in hardware using an LFSR [36] with $g(x)$ as a connection polynomial.

\section{E. Almost XOR-Universal Hash Functions}

In this section we describe almost XOR-universal hash functions which are used in the security analysis in Section IV.

An almost-universal hash function is a keyed function with the property that, for any pair of messages, the probability that the outputs of the hash function on these two messages are equal is small [37]. The probability is taken over the choice of the key and the meaning of "small" usually stated as a parameter of the definition. A slightly stronger variant, an almost XOR-universal hash function, has the property that, for any constant from the output space of the function, the probability for the XOR of the outputs of the hash function on the two inputs is equal to a given constant, is small. Almost-universal and almost XOR-universal hash functions are important information-theoretic tools in cryptography [38], [39].

More formally, almost XOR-universal hash functions are defined as follows. Let $l, n \in \mathbb{N}, K$ be a finite set, and $\epsilon>0$. For a set $S$, we write $A \leftarrow K$ to denote that $A$ is chosen uniformly at random from $K$. In the definitions below, we use capital letters to denote random variables and lower-case letters to denote specific values.

Let $H=\left\{h_{a}:\{0,1\}^{l} \rightarrow\{0,1\}^{n} \mid a \in K\right\}$ be a family of hash functions.

Definition 1:

- $H$ is a universal family of hash functions if $\forall x \neq x^{\prime} \in$ $\{0,1\}^{l}$ :

$$
\operatorname{Pr}_{A \leftarrow K}\left[h_{A}(x)=h_{A}\left(x^{\prime}\right)\right] \leq 2^{-n} .
$$

- $H$ is an $\epsilon$-almost-universal family of hash functions if $\forall x \neq x^{\prime} \in\{0,1\}^{l}$ :

$$
\operatorname{Pr}_{A \leftarrow K}\left[h_{A}(x)=h_{A}\left(x^{\prime}\right)\right] \leq \epsilon .
$$

- $H$ is an $\epsilon$-almost-XOR-universal family of hash functions if $\forall x \neq x^{\prime} \in\{0,1\}^{l}, y \in\{0,1\}^{n}$ :

$$
\operatorname{Pr}_{A \leftarrow K}\left[h_{A}(x) \oplus h_{A}\left(x^{\prime}\right)=y\right] \leq \epsilon .
$$


Krawczyk [40] has shown that a CRC with an irreducible ${ }^{1}$ generator polynomial as the key of the function is an $\epsilon$-almostXOR-universal family of hash functions for $\epsilon=(l+n) / 2^{n-1}$, where $l$ is the message size and $n$ is the CRC size, provided that the CRC is encrypted with a one-time pad of size $n$. In [41] his result was extended to the case of arbitrary reducible polynomials and odd 2 polynomials. The extensions eliminate the need for irreducibility tests, however, have a negative impact on $\epsilon$.

\section{Presented Construction}

The presented construction consists of an $n$-bit LFSR with reconfigurable connections and an $n$-stage APUF (see Figure 3). It takes an $n$-bit seed challenge $C$ as an input and produces an $m$-bit response $R$ as an output.

First, the LFSR is initialized by loading it with a challenge $C$ and clocking the LFSR $n$ times without producing any output. Throughout the paper, we refer to this step as the initialization stage. Assuming the Fibonacci configuration, at each clock step the LFSR state is updated by the state transition function $L: G F\left(2^{n}\right) \rightarrow G F\left(2^{n}\right)$ defined by:

$$
\left(\begin{array}{c}
s_{n-1} \\
s_{n-2} \\
\ldots \\
s_{0}
\end{array}\right) \rightarrow\left(\begin{array}{c}
l\left(s_{0}, \ldots, s_{n-1}\right) \\
s_{n-1} \\
\ldots \\
s_{1}
\end{array}\right) .
$$

where $\left(s_{0}, \ldots, s_{n-1}\right) \in G F\left(2^{n}\right)$ are variables representing values of a current LFSR state and $l: G F\left(2^{n}\right) \rightarrow G F(2)$ is a linear function:

$$
l\left(s_{0}, \ldots, s_{n-1}\right)=\sum_{i=0}^{n-1} g_{i} s_{i}
$$

defined by the LFSR polynomial $g(x)$ :

$$
g(x)=\sum_{i=0}^{n-1} g_{i} x^{i} \oplus x^{n},
$$

where " $\oplus$ " is the $G F(2)$ addition.

Let $S_{i}$ denote the LFSR state at clock step $i, i \in 0,1, \ldots$. Then, during the initialization stage the LFSR goes through the following states:

$$
\begin{aligned}
& S_{0}=C \\
& S_{1}=L(C) \\
& S_{2}=L^{2}(C) \\
& \cdots \\
& S_{n}=L^{n}(C) .
\end{aligned}
$$

The state $S_{n}$ together with the next $m-1$ consecutive LFSR states are used as inputs to the PUF. The PUF generates the following $m$-bit response $R=\left(r_{1}, \ldots, r_{m}\right) \in G F\left(2^{m}\right)$ :

$$
\begin{aligned}
& r_{1}=f\left(L^{n}(C)\right) \\
& r_{2}=f\left(L^{n+1}(C)\right) \\
& \ldots \\
& r_{m}=f\left(L^{n+m-1}(C)\right),
\end{aligned}
$$

\footnotetext{
${ }^{1}$ A polynomial over $G F(2)$ is irreducible if it cannot be factored over $G F(2)$.
}

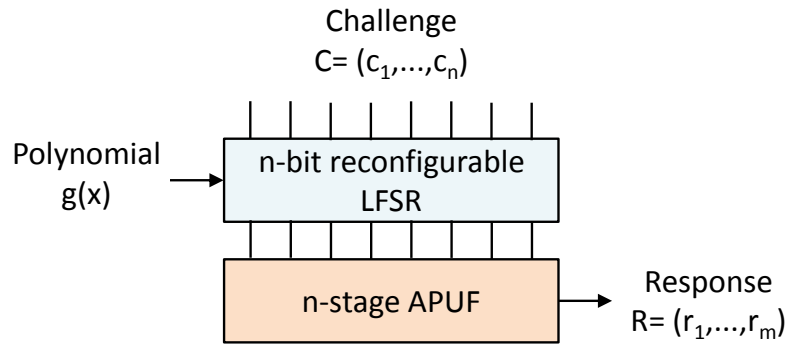

Fig. 3. Block diagram of an $n$-stage CRC-PUF.

where $f: G F\left(2^{n}\right) \rightarrow G F(2)$ is the PUF function defined by (1).

For each new response, the LFSR is reconfigured to a new polynomial $g(x)$ selected uniformly at random from some class of polynomials over $G F(2)$ (e.g. irreducible, odd, or arbitrary) and known to the legitimate parties only.

\section{Security ANALysis}

In this section, we analyze the security of the presented construction.

\section{A. Assumptions}

We assume a typical challenge-response authentication setting in which a verifying party (verifier) and a remote device containing the PUF (prover) communicate over an unsecure channel. The verifier requires the prover to compute the response $R$ to a given challenge $C$. When receiving the correct response $R$, the verifier has assurance that the prover is authentic.

The verifier and the prover share a secret key which is unknown to the attacker. In our case, the key consists of the PUF (for prover)/PUF model (for verifier) and a description of a particular LFSR polynomial $g(x)$ drawn uniformly at random from some class of polynomials over $G F(2)$.

\section{B. Attack Model}

We consider a man-in-the-middle attack scenario in which an attacker can eavesdrop on communication between the verifier and the prover and control the communication by modifying or replacing transmitted messages. The goal of the attacker is to construct the PUF model from the collected challenge-response pairs $(C, R)$.

We assume that the attacker does not have physical access to the PUF, i.e. a physical attack cannot be mounted.

\section{Requirements}

The following requirements should be satisfied in order to use CRC-PUF securely.

1) The challenge $C$ should not be all- 0 .

2) The LFSR polynomial $g(x)$ should be chosen uniformly at random from the set of all possible degree- $n$ polynomials over $G F(2)$ of a given type (irreducible, odd, or arbitrary) for each new response generation session. 


\begin{tabular}{|c|c|c|c|c|}
\hline $\begin{array}{c}\text { Response } \\
\text { size }\end{array}$ & $\begin{array}{c}\text { Challenge } \\
\text { size }\end{array}$ & \multicolumn{3}{|c|}{$\begin{array}{c}\text { Attack success probability } \epsilon \\
\text { for different types of polynomials }\end{array}$} \\
\cline { 3 - 5 } , bits & $n$, bits & Arbitrary [41] & Odd [41] & Irreducible [40] \\
\hline 32 & 32 & $2^{-18.16}$ & $2^{-18.82}$ & $2^{-25.00}$ \\
48 & 48 & $2^{-29.30}$ & $2^{-30.15}$ & $2^{-40.42}$ \\
64 & 64 & $2^{-39.82}$ & $2^{-40.77}$ & $2^{-56.00}$ \\
96 & 96 & $2^{-65.58}$ & $2^{-66.26}$ & $2^{-87.42}$ \\
128 & 128 & $2^{-86.03}$ & $2^{-87.06}$ & $2^{-119.00}$ \\
\hline
\end{tabular}

TABLE I

STATE RECOVERY ATTACK SUCCESS PROBABILITIES FOR DIFFERENT TYPES OF POLYNOMIALS.
3) The challenge and response sizes should be equal, $m=$ $n$. We recommend to use CRC-PUF with the parameters $n=128$ and $m=128$.

The requirement (1) is necessary because, if $C$ is all- 0 , the LFSR is stuck in the all- 0 state. In this case the presented construction generates a response $R$ such that $r_{i}=f(0,0, \ldots, 0)$ for all $i \in\{1, \ldots, m\}$, independently of $g(x)$ and the PUF function $f$. So, the attacker can predict the response to the all-0 challenge with the probability $1 / 2$.

The need for the requirement (2) is explained in the next section.

It is best to use challenges and responses of the same size (requirement (3)) because time complexity of an exhaustive search is $\min \left(2^{n}, 2^{m}\right)$.

\section{Quantitative analysis}

In this section, we estimate security of CRC-PUF quantitatively, as a function of challenge and response sizes.

We consider the case in which the attacker attempts to recover the LFSR state after the initialization stage in order to synchronize challenges with responses.

Theorem 1: If the requirements (1)-(2) are satisfied, the success probability of recovering the LFSR state after the initialization stage is at most $\epsilon$, where $\epsilon$ depends on the type of the LFSR's polynomial, see Table I.

Proof: Loading an $n$-bit LFSR with an $n$-bit challenge $C$ and clocking it $n$ times is equivalent to computing an $n$-bit CRC for the message polynomial $C(x)$ over $G F(2)$ :

$$
C R C_{g}(C)=C(x) \cdot x^{n} \bmod g(x),
$$

where $g(x)$ is the LFSR polynomial. The state bits of the LFSR after $n$ steps are the coefficients of the CRC.

It is known that a CRC with the polynomial $g(x)$ as a key drawn uniformly at random from the set of all possible degree- $n$ polynomials over $G F(2)$ of a given type is an $\epsilon$ almost XOR-universal hash function, provided that the CRC is encrypted with a one-time pad of size $n$. This means that no attacker who does not know $g(x)$ can find the CRC of $C(x)$ with a probability larger than $\epsilon$.

One-time pad encryption is required because:

(a) Since $C R C_{g}(0)=0$ independently of $g(x)$, an attacker can compute a valid CRC for $C(x)=0$ without knowing $g(x)$. (b) Since computation of the CRC is a linear operation, an attacker who knows two pairs $\left(C_{1}, C R C_{g}\left(C_{1}\right)\right)$ and $\left(C_{2}, C R C_{g}\left(C_{2}\right)\right)$ can construct a valid pair $\left(C_{1} \oplus\right.$ $\left.C_{2}, C R C_{g}\left(C_{1}\right) \oplus C R C_{g}\left(C_{2}\right)\right)$ without knowing $g(x)$.

If the requirement (1) is satisfied, then $C(x) \neq 0$ and the attack (a) is prevented. If the requirement (2) is satisfied, then $g(x)$ is updated for each response generation session and the attack (b) is prevented. Therefore, encrypting the CRC with a one-time pad is unnecessary for assuring that the probability of recovering the LFSR state after the initialization stage is at most $\epsilon$.

In the security proof, it is assumed that the polynomial $g(x)$ is drawn uniformly at random. In most practical applications, however, for odd and arbitrary polynomials the coefficients of $g(x)$ will be generated from a secret seed shared by the parties by using, for example, a stream cipher. In this case, the security of the presented construction reduces to the security of the stream cipher in use.

Let us analyze how much information about the PUF model can be extracted if the LFSR state after the initialization is recovered. Consider an example of $n=3$ and $m=n$. If the initial state of the LFSR is $S_{0}=\left(c_{1}, c_{2}, c_{3}\right)$ (where the left-most bit is the LFSR input), then from (4) and (5) we get:

$$
\begin{aligned}
S_{1}= & \left(l(C), c_{1}, c_{2}\right), l(C)=c_{1} g_{2} \oplus c_{2} g_{1} \oplus c_{3} \\
S_{2}= & \left(l^{2}(C), l(C), c_{1}\right), l^{2}(C)=c_{1} g_{2} \oplus c_{2} g_{1} g_{2} \oplus c_{3} g_{2} \\
& \oplus c_{1} g_{1} \oplus c_{2} \\
S_{3}= & \left(l^{3}(C), l^{2}(C), l(C)\right), l^{3}(C)=c_{1} g_{2} \oplus c_{2} g_{1} g_{2} \oplus c_{3} g_{2} \\
& \oplus c_{2} g_{2} \oplus c_{2} g_{1} \oplus c_{3} g_{1} \oplus c_{1}
\end{aligned}
$$

The state $S_{3}$ is used as an input to the PUF to generate the first response bit, $r_{1}$. Similarly we derive equations for the states $S_{4}$ and $S_{5}$ :

$$
\begin{aligned}
S_{4}= & \left(l^{4}(C), l^{3}(C), l^{2}(C)\right), l^{4}(C)=c_{3} g_{2} \oplus c_{2} g_{2} \oplus c_{1} g_{1} g_{2} \\
& \oplus c_{2} g_{1} g_{2} \oplus c_{1} g_{1} \oplus c_{1} g_{2} \oplus c_{3} \\
S_{5}= & \left(l^{5}(C), l^{4}(C), l^{3}(C)\right), l^{5}(C)=c_{2} g_{2} \oplus c_{1} g_{1} g_{2} \oplus c_{3} g_{1} g_{2} \\
& \oplus c_{2} g_{1} \oplus c_{3} g_{1} \oplus c_{3} g_{2} \oplus c_{2}
\end{aligned}
$$

Since $r_{1}=f\left(S_{3}\right), r_{2}=f\left(S_{4}\right), r_{3}=f\left(S_{5}\right)$, from (2) we obtain:

$$
\begin{aligned}
& r_{1}=\operatorname{sgn}\left(w_{1} \Phi_{1}\left(S_{3}\right)+w_{2} \Phi_{2}\left(S_{3}\right)+w_{3} \Phi_{3}\left(S_{3}\right)+w_{4}\right) \\
& r_{2}=\operatorname{sgn}\left(w_{1} \Phi_{1}\left(S_{4}\right)+w_{2} \Phi_{2}\left(S_{4}\right)+w_{3} \Phi_{3}\left(S_{4}\right)+w_{4}\right) \\
& r_{3}=\operatorname{sgn}\left(w_{1} \Phi_{1}\left(S_{5}\right)+w_{2} \Phi_{2}\left(S_{5}\right)+w_{3} \Phi_{3}\left(S_{5}\right)+w_{4}\right)
\end{aligned}
$$

Note that the definition (3) of parity vectors assumes $\{1,-1\}$ encoding of challenges. We can convert it to the binary encoding $\{0,1\}$ as

$$
\begin{aligned}
& \Phi_{i}(C)=\sum_{j=i}^{n}\left(1-2(-1)^{c_{j}}\right), \text { for } 1 \leq i \leq n, \\
& \Phi_{n+1}=1 .
\end{aligned}
$$

where $c_{j} \in\{0,1\}$. After substituting these expressions of the parity vectors into (7), we get: 


$$
\begin{aligned}
r_{1} & =w_{1}\left(3-2(-1)^{l^{3}(C)}-2(-1)^{l^{2}(C)}-2(-1)^{l(C)}\right) \\
& +w_{2}\left(2-2(-1)^{l^{2}(C)}-2(-1)^{l(C)}\right) \\
& +w_{3}\left(1-2(-1)^{l(C)}\right)+w_{4} \\
r_{2} & =w_{1}\left(3-2(-1)^{l^{4}(C)}-2(-1)^{l^{3}(C)}-2(-1)^{l^{2}(C)}\right) \\
& +w_{2}\left(2-2(-1)^{l^{3}(C)}-2(-1)^{l^{2}(C)}\right) \\
& +w_{3}\left(1-2(-1)^{l^{2}(C)}\right)+w_{4} \\
r_{3} & =w_{1}\left(3-2(-1)^{l^{5}(C)}-2(-1)^{l^{4}(C)}-2(-1)^{l^{3}(C)}\right) \\
& +w_{2}\left(2-2(-1)^{l^{4}(C)}-2(-1)^{l^{3}(C)}\right) \\
& +w_{3}\left(1-2(-1)^{l^{3}(C)}\right)+w_{4}
\end{aligned}
$$

We described $r_{1}, r_{2}, r_{3}$ as functions of $g_{1}, g_{2}$ and $w_{1}, w_{2}, w_{3}, w_{4}$. According to Theorem 1 , with the probability at most $\epsilon$ the attacker may succeed to recover the state $S_{3}$. Note that this does not immediately imply that the attacker gets $g(x)$, the success probability depends on the polynomial type. If the attacker can recover $g(x)$, he/she can substitute the coefficients of $g(x)$ into (8) and get linear inequalities. For example, if $\left(c_{1}, c_{2}, c_{3}\right)=(1,1,1)$ and $g_{1}=1, g_{2}=0$ then $l(C)=0, l^{2}(C)=0, l^{3}(C)=1, l^{4}(C)=0, l^{5}(C)=1$. Suppose that $r_{1}=0, r_{2}=1, r_{3}=1$. Then (8) reduces to

$$
\begin{aligned}
& w_{1}-2 w_{2}-w_{3}+w_{4}<0 \\
& w_{1}+2 w_{2}-w_{3}+w_{4} \geq 0 \\
& 5 w_{1}+2 w_{2}+3 w_{3}+w_{4} \geq 0
\end{aligned}
$$

From these linear inequalities the attacker may be able to extract some partial information about the weights, e.g. from the 1st and 2nd inequalities it can be concluded that

$$
4 w_{2} \geq 0 .
$$

However, clearly it is not possible to deduce values of $n+1$ weights by observing only one $n$-bit response. To do this, the attacker needs to see more responses and, to get them, he/she needs to perform more than one successful state recovery attack. Let $k$ be the minimal number of $m$-bit responses required to deduce all $n+1$ weights. If the LFSR polynomial is selected uniformly at random for every new response, the probability of $k$ successful the state recovery attacks is $\epsilon^{k}$. From Table I we can see that, for any $k>1, \epsilon^{k}<2^{-n}$ for all polynomial types. Therefore, performing more than one successful state recovery attack is slower than exhaustive response search.

We applied the classical LR-based modeling attack [3] implemented in the Python PUF simulator and learner package (https://github.com/nils-wisiol/pypuf) to test with which accuracy a 128-stage CRC-PUF model can be learned from a single 128-bit response $R$ if the attacker knows the LFSR polynomial $g(x)$. The results showed that the prediction accuracy is at most $75 \%$ per bit. So, the prediction accuracy for 128 bits is at most $(0.75)^{128} \approx 2^{-53}$. Therefore, for a 128-bit CRC-PUF, the probability that an attacker correctly predicts the response for any challenge that has not been used in the past is at most $2^{-86} \times 2^{-53}=2^{-139}$. Again, this is slower than exhaustive response search.

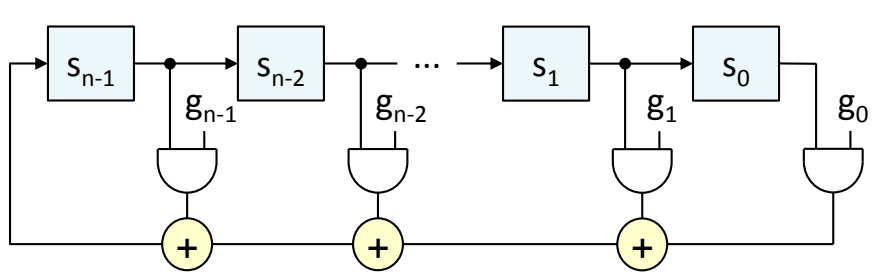

Fig. 4. Block diagram of an $n$-bit LFSR with reconfigurable connections.

It does not seem possible to apply a reliability-based modeling attack [32] to the presented construction. Since the LFSR polynomial is changed for each session, different responses are generated by CRC-PUF for the same challenge. Thus, it is not clear how the extracted reliability information can be used.

\section{HARDWARE COST ANALYSIS}

In the presented construction, security is assured by changing the LFSR polynomial for every response. This is implemented by using an LFSR with reconfigurable connections, see Fig. 4. Techniques for implementing reconfigurable LFSRs are well-known. Reconfigurable LFSRs are used, for example, in applications which support multiple CRC standards [42].

Note that other ML attack resistant APUF-based constructions, including $k$-XOR PUF [21] and interpose PUF [23], also use LFSRs for expanding a seed challenge into a sequence of $m$ challenges in order to reduce data transfer between a verifier and a prover. However, their LFSR's polynomials are fixed. The overhead of using a reconfigurable $n$-bit LFSR is only $n$ 2-input AND gates and at most $n-12$-input XOR gates. This is more efficient than, for example, adding another $n+1$-stage APUF to the construction, as in interpose PUF case, or $k-1 n$-stage APUFs, as in $k$-XOR PUF case.

Table III compares CRC-PUF to other types of PUFs in terms of Gate Equivalent (GE). ${ }^{2}$ Note that a 128-bit APUF is not ML attack resistant. It is included in the table as a base reference. The number $4.1 \mathrm{~K} \mathrm{GE}$ for the ising PUF is taken from [24] where a 64-input ising PUF design is evaluated. Other numbers are derived from the PUF's circuit diagrams using the gate parameters specified in Table II.

It is also worth mentioning that a $65 \mathrm{~nm}$ CMOS implementation of a 128-bit LFSR with reconfigurable connections (Figure 4) has $2067 \mathrm{GE}$, while a 65nm CMOS implementation of a 128-bit SHA-3 hash function [26] has $34743 \mathrm{GE}$, which is 16.8 times more. Therefore, using a full-strength cryptographic hash function instead of the CRC-based hash would require considerably more resources.

Note that the presented analysis does not take into account the complexity of generating LFSR polynomials. As we mentioned in Section IV-D, in most practical applications the coefficients of $g(x)$ will be generated using e.g. a stream cipher from a secret seed shared by the parties. So, if a stream cipher

\footnotetext{
${ }^{2} \mathrm{~A}$ gate equivalent is a technology-independent unit of measure of circuit area. For CMOS technology, the area of a smallest two-input NAND gate in the library is usually used as gate equivalent.
} 


\begin{tabular}{|c|c|}
\hline Gate & Area, GE \\
\hline 2-input NAND & 1 \\
2-input AND & 1.5 \\
2-input XOR & 2.5 \\
2-to-1 MUX & 2.5 \\
flip-flop & 6.25 \\
\hline
\end{tabular}

TABLE II

PARAMETERS OF GATES AND FLIP-FLOPS FOR A TYPICAL 65 NM CMOS TECHNOLOGY; GE = GATE EQUIVALENT.

\begin{tabular}{|c|c|c|c|}
\hline$n$ & PUF type & GE & GE PUF \\
\hline 128 & APUF [21] & 646 & 1 \\
128 & 8-XOR-APUF [21] & 5144 & 7.96 \\
128 & interpose PUF [23] & 1291 & 1.99 \\
64 & ising PUF [24] & 4100 & 6.34 \\
128 & CRC-PUF & 1156 & 1.78 \\
\hline
\end{tabular}

TABLE III

COMPARISON OF CRC-PUF WITH OTHER PUFS IN TERMS OF AREA.

is available in the system, the generation of LFSR polynomials does not bring much extra overhead.

\section{Statistical Analysis}

In this section, we evaluate statistical properties of CRCPUF for the parameters $n=128$ and $m=128$ by simulation. Delay variations of switch blocks are assumed to follow a Gaussian distribution with standard deviation of $10 \%$. This is a standard assumption for CMOS APUF implementations [21].

\section{A. Uniformity}

The first important metric to evaluate is uniformity, which reflects the proportion of ' 0 's and ' 1 's in the responses.

The uniformity is quantified using the Hamming Weight $H W$, of responses:

$$
H W(R)=\sum_{i=1}^{n} b_{i}
$$

where $b_{i}$ is the value of the $i$ th bit of the response $R$ and $n$ is the number of bits in $R$. The probability of ' 1 's in $k n$-bit responses $R_{i}, P_{1}$, is given by the average HW:

$$
P_{1}=\frac{1}{k} \sum_{i=1}^{k} \frac{H W\left(R_{i}\right)}{n} \times 100 \% .
$$

Figure 5 shows the distribution of $H W$ of 10.000128 bit responses derived from 10.000 instances. The average $H W$ is 64.0995 , which is close to the ideal value $n / 2$. This corresponds to $P_{1}=50.0777 \%$.

\section{B. Uniqueness}

Uniqueness is another important metric, which characterizes how easily one PUF instance can be distinguished from another PUF instance.

The uniqueness is quantified using the Hamming Distance, $H D$, between $n$-bit responses $R_{i}$ and $R_{j}$ of two different PUF instances:

$$
H D\left(R_{i}, R_{j}\right)=\sum_{k=1}^{n}\left(a_{k} \oplus b_{k}\right)
$$

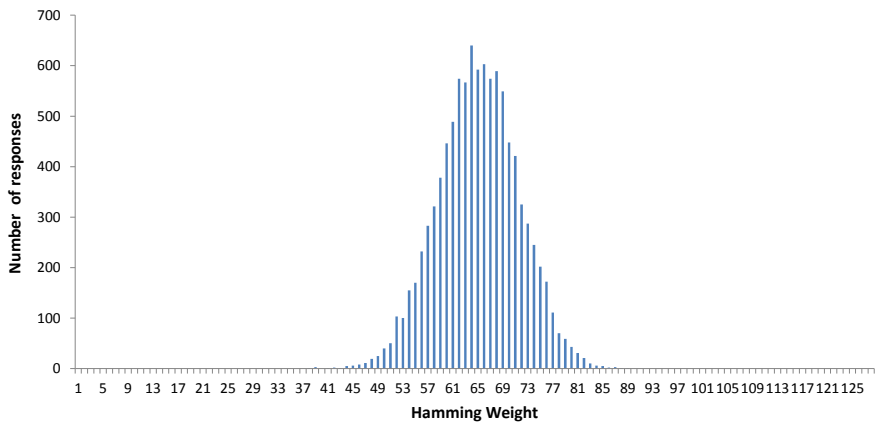

Fig. 5. Distribution of Hamming weight of 10.000 128-bit responses obtained from 10.000 CRC-PUF instances.

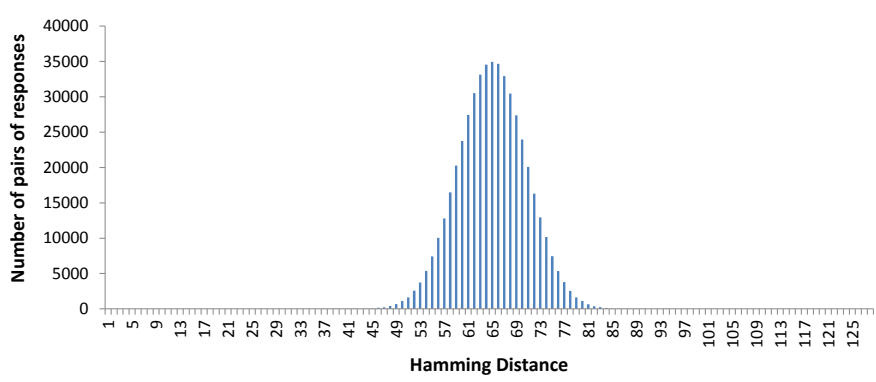

Fig. 6. Distribution of Hamming distance of 499.500 pairs of 128-bit responses obtained from 1.000 CRC-PUF instances.

where $a_{k}$ and $b_{k}$ are the values of the $k$ th bits of the responses $R_{i}$ and $R_{j}$, respectively.

The uniqueness is calculated based on the average $H D$ of pairs of responses of different PUF instances as follows:

$$
U=\frac{2}{k(k-1)} \sum_{i=1}^{k-1} \sum_{j=i+1}^{k} \frac{H D\left(R_{i}, R_{j}\right)}{n} \times 100 \%
$$

where $k$ is the total number of PUF instances and $R_{i}$ and $R_{j}$ are the $n$-bit responses of PUF instances $i$ and $j$, respectively. For perfect identification, the average $H D$ between responses of different PUFs should be $n / 2$. This corresponds to $U=$ $50 \%$.

We derived 128-bit responses from $k=1.000$ instances. To compute the average $H D, 1000 \times 999 / 2=499.500$ evaluations of $H D\left(R_{i}, R_{j}\right)$ were made in total. Figure 6 shows the distribution of $H D$ for all pairs. The average $H D$ is 63.9971 , which is close to the ideal value $n / 2$. The corresponding uniqueness is $U=49.9978 \%$.

\section{CONCLUSION}

In this paper, we introduced a new lightweight PUF construction CRC-PUF based on an arbiter PUF and an LFSR with reconfigurable connections. Our experimental results show that the presented construction can be efficiently implemented and has good statistical properties. At the same time, it appears to be free of well-known weakness of the arbiter PUF to modeling attacks. These conditions are only minimal necessary conditions for the presented construction to deserve attention 
and obviously does not guarantee that it cannot be broken. Its practical strength can be determined only after a public evaluation.

In future work, we plan to evaluate the resistance of CRCPUF to physical attacks such as invasive attacks [43], sidechannel analysis [44], and fault injection [45].

\section{ACKNOWLEDGMENT}

This work was supported in part by the research grants 2017-05232 and 2018-03964 from VINNOVA, and 201804482 from the Swedish Research Council.

\section{REFERENCES}

[1] D. Freeman and C. Chio, Machine Learning and Security. O'Reilly Media, 2018.

[2] B. B. Gupta and Q. Z. Sheng, Machine Learning for Computer and Cyber Security: Principle, Algorithms, and Practices. CRC Press, 2019.

[3] U. Rührmair, F. Sehnke, J. Sölter, G. Dror, S. Devadas, and J. Schmidhuber, "Modeling attacks on physical unclonable functions," in Proc. of the 17th ACM Conference on Computer and Communications Security, CCS '10, (New York, NY, USA), pp. 237-249, ACM, 2010.

[4] A. Heuser and M. Zohner, "Intelligent machine homicide," in Constructive Side-Channel Analysis and Secure Design (W. Schindler and S. A. Huss, eds.), pp. 249-264, 2012.

[5] T. Bartkewitz et al , "Efficient template attacks based on probabilistic multi-class support vector machines," in Smart Card Research and Advanced Applications, pp. 263-276, 2013.

[6] L. Lerman, G. Bontempi, and O. Markowitch, "Power analysis attack: An approach based on machine learning," vol. 3, 012014.

[7] H. Maghrebi et al, "Breaking cryptographic implementations using deep learning techniques," in 6th Int. Conf. on Security, Privacy, and Applied Cryptography Engineering, pp. 3-26, 122016.

[8] L. Huang, A. D. Joseph, B. Nelson, B. I. Rubinstein, and J. D. Tygar, "Adversarial machine learning," in Proceedings of the 4th ACM Workshop on Security and Artificial Intelligence, pp. 43-58, ACM, 2011.

[9] S. Bengio, I. J. Goodfellow, and A. Kurakin, "Adversarial machine learning at scale," in Int. Conference on Learning Representations, 2017.

[10] A. D. Joseph, B. Nelsson, B. Rubinstain, and J. Tygar, Adversarial Machine Learning. Cambridge University Press, 2017.

[11] Y. Vorobeychik, M. Kantarcioglu, R. Brachman, P. Stone, and F. Rossi, Adversarial Machine Learning. Synthesis Lectures on Artificial Intelligence and Machine Learning, Morgan \& Claypool Publishers, 2018.

[12] B. Biggio, B. Nelson, and P. Laskov, "Support vector machines under adversarial label noise," in Proceedings of the Asian Conference on Machine Learning (C.-N. Hsu and W. S. Lee, eds.), vol. 20 of Proceedings of Machine Learning Research, (South Garden Hotels and Resorts, Taoyuan, Taiwain), pp. 97-112, PMLR, 14-15 Nov 2011.

[13] S. Chen, M. Xue, L. Fan, S. Hao, L. Xu, H. Zhu, and B. Li, "Automated poisoning attacks and defenses in malware detection systems: An adversarial machine learning approach," Computers \& Security, vol. 73 , pp. $326-344,2018$.

[14] R. Pappu, B. Recht, J. Taylor, and N. Gershenfeld, "Physical one-way functions," Science, vol. 297, no. 5589, pp. 2026-2030, 2002.

[15] D. Lim, J. W. Lee, B. Gassend, G. E. Suh, M. van Dijk, and S. Devadas, "Extracting secret keys from integrated circuits," IEEE Transactions on Very Large Scale Integration (VLSI) Systems, vol. 13, pp. 1200-1205, Oct 2005.

[16] P. Tuyls, G.-J. Schrijen, B. Škorić, J. van Geloven, N. Verhaegh, and R. Wolters, Read-Proof Hardware from Protective Coatings, pp. 369383. Berlin, Heidelberg: Springer Berlin Heidelberg, 2006.

[17] S. Chellappa and L. T. Clark, "SRAM-based unique chip identifier techniques," IEEE Transactions on Very Large Scale Integration (VLSI) Systems, vol. 24, pp. 1213-1222, April 2016.

[18] S. Skorobogatov, "Physical attacks and tamper resistance," in Introduction to Hardware Security and Trust (M. Tehranipoor and C. Wang, eds.), Inf. Security and Cryptography, Springer Berlin / Heidelberg, 2011.

[19] I. Krsti, "Behind the Scenes with iOS Security," Aug. 2016.

[20] J. Tobisch and G. T. Becker, On the Scaling of Machine Learning Attacks on PUFs with Application to Noise Bifurcation, pp. 17-31. Cham: Springer International Publishing, 2015.
[21] D. Lim, "Extracting secret keys from integrated circuits," Master's thesis, Massachusetts Institute of Technology, 2004.

[22] M. Majzoobi, F. Koushanfar, and M. Potkonjak, "Testing techniques for hardware security," in 2008 IEEE International Test Conference(ITC), vol. 00, pp. 1-10, Oct. 2009.

[23] P. H. Nguyen, D. P. Sahoo, C. Jin, K. Mahmood, U. Rhrmair, and M. van Dijk, "The interpose PUF: Secure PUF design against state-of-the-art machine learning attacks." Cryptology ePrint Archive, Report 2018/350, 2018.

[24] H. Awano and T. Sato, "Ising-PUF: A machine learning attack resistant PUF featuring lattice like arrangement of arbiter-PUFs," in 2018 Design, Automation Test in Europe Conference Exhibition (DATE), pp. 14471452, March 2018.

[25] B. Gassend, M. V. Dijk, D. Clarke, E. Torlak, S. Devadas, and P. Tuyls, "Controlled physical random functions and applications," ACM Trans. Inf. Syst. Secur., vol. 10, pp. 3:1-3:22, Jan. 2008.

[26] M. J. Dworkin, "Sha-3 standard: Permutation-based hash and extendable-output functions." NIST Pub Series: Federal Inf. Process. Stds., Report 202, 2015.

[27] R. Maes, Physically Unclonable Functions: Constructions, Properties and Applications. Springer, 2013.

[28] C. Cannière and B. Preneel, "Trivium," New Stream Cipher Designs: The eSTREAM Finalists, LNCS 4986, pp. 244-266, 2008.

[29] D. Sahoo, P. Nguyen, D. Mukhopadhyay, and R. Chakraborty, "A case of lightweight PUF constructions: Cryptanalysis and machine learning attacks," Computer-Aided Design of Integrated Circuits and Systems, IEEE Transactions on, vol. 34, pp. 1334-1343, 082015.

[30] L. G. Valiant, "A theory of the learnable," Commun. ACM, vol. 27, pp. 1134-1142, Nov. 1984.

[31] F. Ganji, S. Tajik, and J.-P. Seifert, "PAC learning of arbiter PUFs," Journal of Cryptographic Engineering, vol. 6, pp. 249-258, Sep 2016.

[32] G. Becker, "The gap between promise and reality: On the insecurity of XOR arbiter PUFs," pp. 535-555, 2015.

[33] N. Wisiol, C. Graebnitz, M. Margraf, M. Oswald, T. Soroceanu, and B. Zengin, "Why attackers lose: Design and security analysis of arbitrarily large XOR arbiter PUFs," in PROOFS 2017. 6th International Workshop on Security Proofs for Embedded Systems.

[34] E. Dubrova, Fault-Tolerant Design. Springer, 2013.

[35] T.-B. Pei and C. Zukowski, "High-speed parallel CRC circuits in VLSI," IEEE Transactions on Communications, vol. 40, pp. 653 -657, Apr. 1992.

[36] S. Golomb, Shift Register Sequences. Aegean Park Press, 1982.

[37] D. R. Stinson, "Universal hashing and authentication codes," Des. Codes Cryptography, vol. 4, pp. 369-380, Oct. 1994.

[38] D. R. Stinson, "On the connections between universal hashing, combinatorial designs and error-correcting codes," in In Proc. Congressus Numerantium 114, pp. 7-27, 1996.

[39] H. Tyagi and A. Vardy, "Universal hashing for information-theoretic security," Proceedings of the IEEE, vol. 103, pp. 1781-1795, Oct 2015.

[40] H. Krawczyk, "LFSR-based hashing and authentication," in Proceeding of the 14th Annual International Cryptology Conference on Advances in Cryptology, CRYPTO '94, pp. 129-139, Springer-Verlag, 1994.

[41] E. Dubrova, M. Näslund, G. Selander, and F. Lindqvist, "Message authentication based on cryptographically secure CRC without polynomial irreducibility test," Cryptography and Communications, vol. 10, pp. 383399, Mar 2018.

[42] J. Birch, L. G. Christensen, and M. Skov, "A programmable $800 \mathrm{Mbit} / \mathrm{s}$ CRC check/generator unit for LANs and MANs," Comput. Netw. ISDN Syst., vol. 24, pp. 109-118, Apr. 1992.

[43] S. Tajik, E. Dietz, S. Frohmann, J.-P. Seifert, D. Nedospasov, C. Helfmeier, C. Boit, and H. Dittrich, "Physical characterization of arbiter PUFs," in Cryptographic Hardware and Embedded Systems (CHES'2014), pp. 493-509, 2014.

[44] J. Delvaux and I. Verbauwhede, "Side channel modeling attacks on 65nm arbiter PUFs exploiting CMOS device noise," in Proc. of IEEE International Symposium on Hardware-Oriented Security and Trust (HOST'2013), pp. 137-142, 062013.

[45] J. Delvaux and I. Verbauwhede, "Fault injection modeling attacks on $65 \mathrm{~nm}$ arbiter and RO sum PUFs via environmental changes," IEEE Transactions on Circuits and Systems I: Regular Papers, vol. 61, pp. 1701-1713, 062014. 